\title{
Exosome Extracellular Vesicles: A Vehicle for Simultaneous Immune and Genetic Therapy
}

\section{Phillip W Askenase}

Section of Rheumatology and Clinical Immunology, Department of Internal Medicine, Yale University School of Medicine, USA

${ }^{\star}$ Corresponding author: Phillip W Askenase, Section of Rheumatology and Clinical Immunology, Department of Internal Medicine, Yale University School of Medicine, 333 Cedar Street, New Haven Connecticut, 06520, USA, E-mail: philip.askenase@yale.edu

Received: April 05, 2020; Accepted: April 15, 2020; Published: April 20, 2020

\section{Short Review}

Exosomes are newly recognized universal minute nanosize particles made by all cells in all species that transfer genetic instructions between cells. They additionally can be made immune specific by antibody coating to achieve dual specific antigen targeting of particular acceptor cells, as well as being vehicles to deliver genetic information as RNAs to alter targeted cell function. Because of exceptional resistance properties, some therapeutic exosomes can be administered orally.

\section{What are exosomes?}

Exosomes are minute nano-sized lipid sacs called vesicles that are produced and then secreted by all cell types in all animal species. They are a sensational biologic discovery. As universal nano-particles of life, they are very small, but a big thing since they seem to be involved in nearly all biological and clinical processes. Importantly, these Nanovesicles may lead to new and highly advantageous therapies. Exosomes are the most common subset among a large very diverse group that exists outside of cells called extracellular vesicles. Exosomes are tiny spheres with an average diameter of 100 Nano meters, or about one hundredth the sizes of the producing cells (Figure 1). Production and release of extracellular vesicles, occurs in all animals, plants, fish, fungi and also in the basic single cell forms of life; such as bacteria and even most primitive archaea (Figure 2).

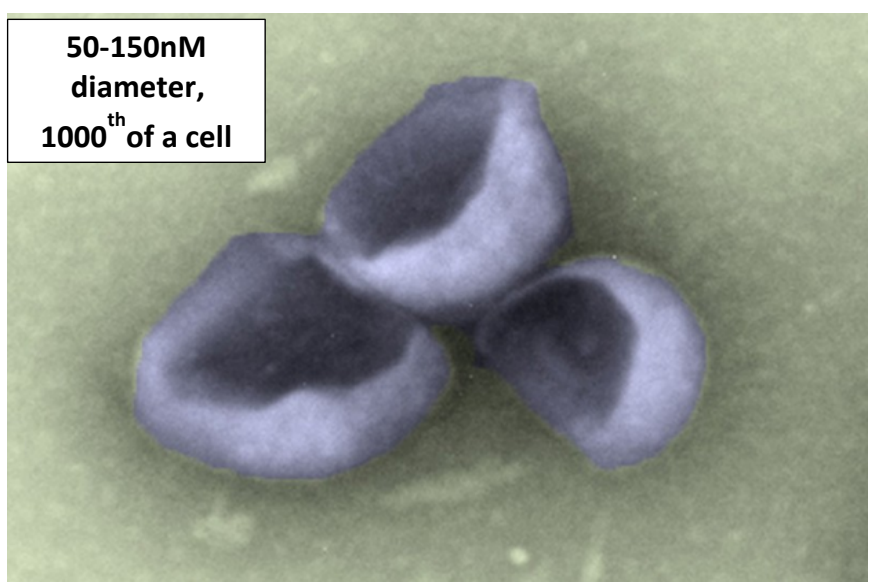

Figure 1. Scanning electron microscopy of individual exosome that are spheres in their native state but here distorted in processing.

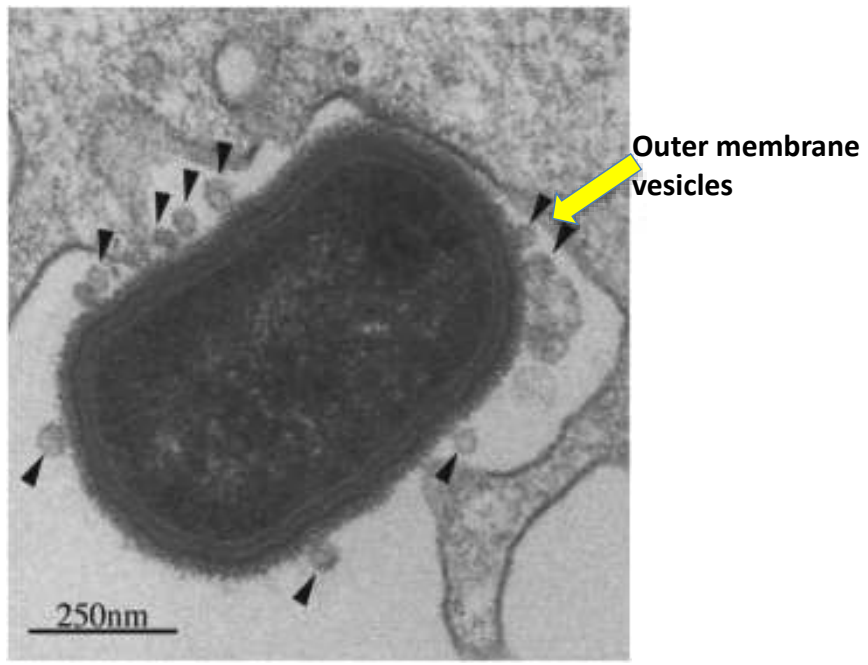

Figure 2. Staphylococcus aureus outer membrane exosome-like vesicles pinched out from the surface of the bacteria.'

\section{The exosome nano world}

Therefore, exosomes and other diverse extracellular vesicles from many cell types are present in all body fluids and can be described as a cloud of mixed nano particles between cells (Figure 4). In humans and mice, exosomes in the peripheral blood are about a billion per milliliter compared to white blood cells that are present at ten thousand per milliliter. Among the myriad of extracellular vesicle subsets, exosomes have been show to alter functions in targeted cells. The main and entirely new biologic function of exosomes is their ability to enter other acceptor cells, near or far via the blood stream, in order to transfer the genetic-acting molecules they carry [1-5].

In particular, they transfer micro RNAs (miRNAs) that are small ribonucleotide polymers of only about 22 base pairs (Figure 3 ). These exosomes carry and transfer extracellular miRNAs can produce modifications of the DNA in the nucleus of the acceptor cells to alter their genetic mediated production of proteins that in turn alters target cell function. In sum, exosomes are a completely unanticipated nano entity that can mediate entirely new biological processes, and alter molecular and metabolic pathways of acceptor cells. As such, they are likely involved in many diseases. 


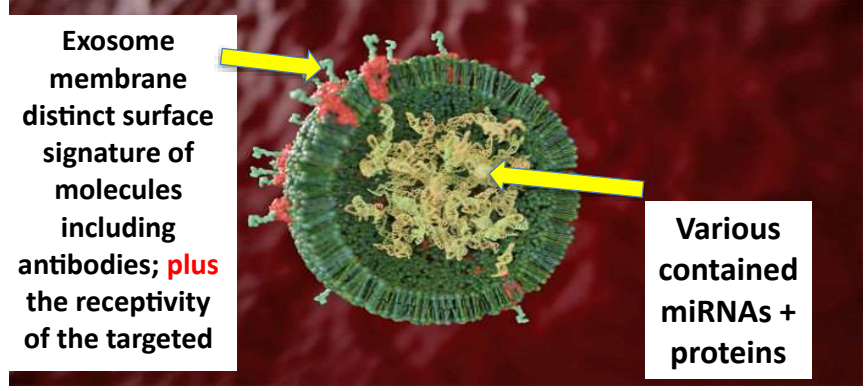

Figure 3. Cross section of an individual idealized exosome showing surface signature of various adhesion molecules that can include antigen specific antibodies. These respectively can mediate semi-specific binding to comparable receptors on the surface of acceptor cells, or antigen specific targeting of acceptor cells. Inside the exosome are various RNAs, including miRNAs and proteins.

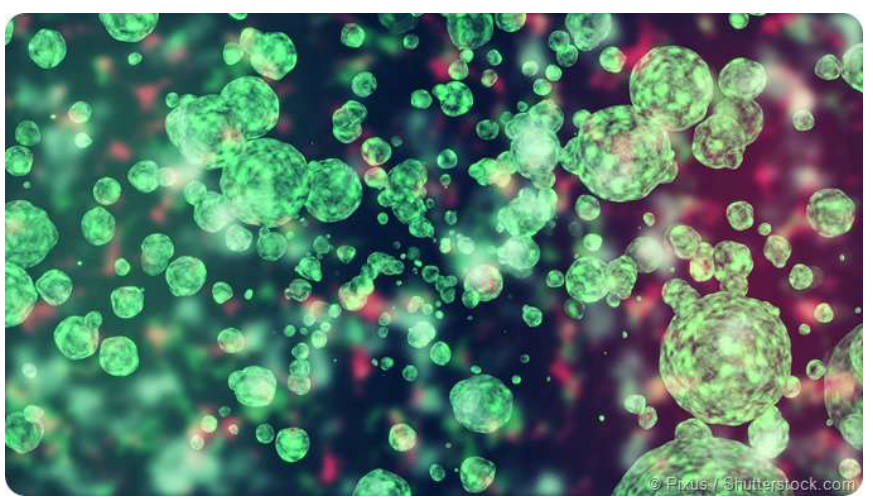

Figure 4. Intercellular tissue cloud of various extracellular vesicles between the cells, of which some are secreted exosomes.

\section{Clinical consequences}

As the dominant mechanism for inter cellular transfer of genetic function, exosomes have great medical importance. They introduce many new possibilities; such as: better understanding of disease mechanisms, new ways for making diagnoses after isolation from blood and body fluids (so called liquid biopsies) and then analyzed molecularly, and as vehicles for new natural nano vesicle therapies. Appropois of this last point they have great advantages over the current numerous problems associated with designing artificial nano therapeutic particles that lack specificity, are unable to cross tissue barriers, and are rapidly eliminated by natural mechanisms that detect their artificiality.

\section{Exosome therapies}

Exosomes are a very promising therapy because of their very small size, their ability to cross natural tissue barriers such as those that protect the brain, and when administered into the circulation have prolonged life over days. This is because they are natural nanovesicles that are able to avoid host cells that remove the artificial particles. Compared to cells used for therapy, exosomes have unusual stability; resistance to noxious environments, and long storage ability of biologically active genetic contents. Exosome vesicles are a physiological natural system for delivering genetic and antiinflammatory molecules; thus constituting new treatment modalities for a variety of diseases.
Exosomes can be isolated from healthy individuals and easily enriched for delivery to individuals with a disease. Further, exosomes in some instances can be used across species, or even from plants can be used without concern for immunologic or genetic incompatibility, since the miRNAs are often universal across species. They usually contain no DNA and thus are without danger of transformation to cancers; compared to therapies with cells. New work in a variety of fields indicates that exosomes may be effective therapy for cancers, arthritis, stroke, spinal cord injury, myocardial infarction, lung fibrosis, and other diseases. Also, investigations have begun in autoimmune conditions, such as multiple sclerosis, and in degenerative conditions, such as Alzheimer's or Parkinson's diseases, and even in autism.

\section{Exosomes have unusual durability, stability and ability to resist harsh conditions}

Unlike cells, exosomes have special membranes composed of unusual proportions of lipid components, resulting in high surface viscosity and rigidity. This enables them to resist harsh conditions that cells cannot survive. These properties of resistance are postulated to be derived from their ancient origins near the beginning of biologic evolution. Some current exosomes are proposed as being related to the "pro cells" from that primordial era that existed before the development of bacteria.

Other unusual related properties of some current day exosomes, that are derived from activated immune cells, include the ability to bind antigen-specific antibody chains on their surface, and further accept added selected miRNAs. Together, these abilities can achieve unprecedented combined immune antigen-specific cell targeting via the surface antibodies binding the acceptor cells, as well as subsequent exosome delivery of particular gene-altering functional miRNAs.

\section{Exosomes in milk survive harsh conditions of gastric digestion}

Mothers breast milk is an outstanding example of the strong resistance of exosomes to harsh conditions. Milk is loaded with exosomes carrying diverse and unusual miRNAs, and have strong resistance to the noxious environment in the neonatal stomach. This consists of the combined actions of a variety of digestive enzymes in high acidity. Surviving this noxious gastric environment, the breast milk exosomes can be intestinally absorped by the neonate for subsequent transfer of their miRNAs to potentially regulate a variety of developing systems.

\section{Successful therapy with exosomes; including dual immune and gene specific oral treatment}

The unusual property of resistance to stomach degradation can allow oral administration of therapeutic exosomes to patients. These natural nano-vesicles can be constructed to be immune Ag-specific suppressive exosomes by surface sbinding of chosen antibody for specific acceptor cell targeting. Further, these exosomes can be constructed to carry chosen particular inhibitory miRNAs. After intestinal absorption, such exosomes can strongly suppress immune inflammatory antigen-specific $\mathrm{T}$ cell allergy responses in the skin of recipients for several days, as demonstrated in Figure 5 


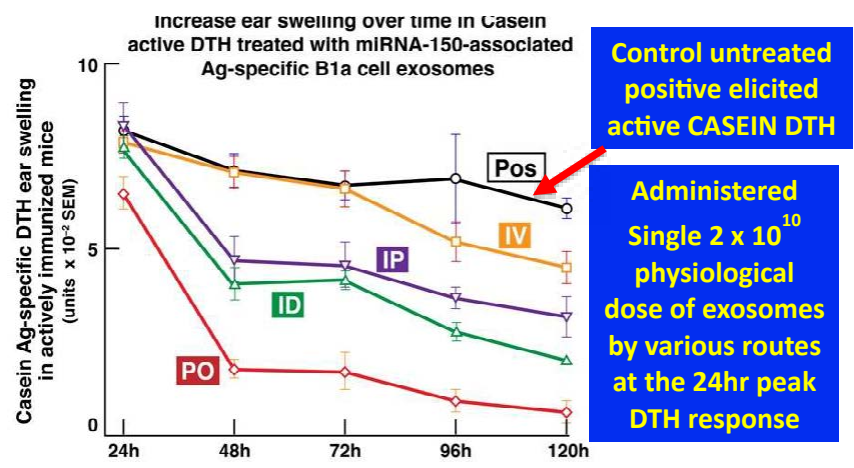

Figure 5. The figure shows experiments with milk protein (casein) allergy, expressed as swelling in the ear skin of mice following local injection. Systemic treatments with exosomes from suppressor $\mathrm{T}$ cells were begun at the 24 hour height of the positive ear swelling response (black line). These immune allergic tissue swelling responses and were strongly inhibited for the subsequent four days by administration of exosomes that were casein antigen-specific via surface antibody chains, and miRNA-150 transferring. They were adminisered by several routes, and were most strongly inhibitory when given orally (PO, red line), compared to the usually employed systemic routes of administration; such as intravenous (IV, orange line), or into the peritoneum (IP, purple line), or subcutaneous at a skin site distant from the ears (intradermal, ID, green line).

The oral route of administration has been superior (black line) compared to the usual intravenous, intraperitoneal and subcutaneous routes. This evidence suggests that oral treatment is a more physiological route of administration, compared to the usual injection routes. To our knowledge, such chosen dual antigen-specific and selected miRNA mediated gene expression altering exosome therapy has not been achieved previously. The prospect of efficacious oral administration would undoubtedly have greater patient acceptance and comfort, especially in children. For example, in treatment of cancers, exosome's ability to target specific cells and regulate protein synthesis in combination with chemotherapy and radiotherapy, likely will allow their reduced dose which in turn lessens toxicity of these existing therapeutic approaches. (1114 words)

\section{Acknowledgements}

The author is grateful for grant support from the NIH, the hard laboratory work of Krzysztof Bryniarski and Katarzyna Nazimek who produced the quoted and presented data. I am particularly indebted to Dr. Irwin Braverman for his thorough review and recommendations.

\section{References}

1. Krzysztof Bryniarski, Wlodzimierz Ptak, Emilia Sikora, Katarzyna Nazimek, et al (2018) Free extracellular miRNA functionally targets cells by transfecting exosomes from their companion cells, PLoS One 10:e122991.

2. Bryniarski K, Ptak W, Jayakumar A, Tuschl T, Hafner M, Püllmann K, et al. (2013) Antibody light chain coated antigen-specific exosomes deliver suppressor $\mathrm{T}$ cellderived miRNA-150 to inhibit effector T cells. J Allergy Clin Immunol 132:170-81.

3. Krzysztof Bryniarski, Katarzyna Nazimek, Wlodzimierz Ptak, Tom Groot Kormelink, and Philip W Askenase (2020) Orally Administered T and B cell Antigen-Specific Suppressor Exosomes Deliver miRNA-150 to Inhibit DTH Via Their Surface Antibody Light Chains Binding Antigen Peptides in MHC on APC Targeted Cells.

4. Magdalena Wąsik, Katarzyna Nazimek, Bernadeta Nowak, Philip W Askenase, and Krzysztof Bryniarski (2019) Delayed-Type Hypersensitivity Underlying Casein Allergy Is Suppressed by Extracellular Vesicles Carrying miRNA-150. Nutrients 11:9

5. Carolina de la Torre Gomez, Renee V Goreham, Joan J Bech Serra, Thomas Nann and Martin Kussmann (2018) "Exosomics"-A Review of Biophysics, Biology and Biochemistry of Exosomes With a Focus on Human Breast Milk. Front Genet 27.

6. Kamerkar S, LeBleu V, Sugimoto H. et al. (2017) Exosomes facilitate therapeutic targeting of oncogenic KRAS in pancreatic cancer. Nature 546: 498-503.

7. Jeppesen DK, Fenix AM, Franklin JL, et al. (2019) Reassessment of Exosome Composition. Cell 177:428-445.e18.

8. Sukhvinder Gill, Ryan Catchpole and Patrick Forterre (2019) Extracellular membrane vesicles in the three domains of life and beyond. FEMS Microbiology Reviews 43: 273-303

9. Carmen Schwechheimer and Meta J Kuehn (2015) Outer-membrane vesicles from Gram-negative bacteria: biogenesis and functions. Nat Rev Microbiol13:605-19.

10. Jack W Szostak (2016) On the origin of life. MEDICINA (Buenos Aires) 76: 199-203

11. Raposo G, Stoorvogel W (2019) Extracellular vesicles: exosomes, microvesicles, and friends. J Cell Biol 200:373-383.

12. Clotilde Théry (2011) Exosomes: secreted vesicles and intercellular communications. F1000 Biol Rep 3:15.

\section{Citation:}

Phillip W Askenase (2020) Exosome Extracellular Vesicles: A Vehicle for Simultaneous Immune and Genetic Therapy Microbiol Immunol Pathol Volume 2(1): 1-3. 